Arthroskopie 2013 $\cdot 26: 250-250$

DOI 10.1007/s00142-013-0758-8

Online publiziert: 23. Oktober 2013

(c) Springer-Verlag Berlin Heidelberg 2013

P. Lobenhoffer ${ }^{1}$. S. Rupp ${ }^{2}$

${ }^{1}$ Sportsclinic Germany, Hannover

${ }^{2}$ Fachklinik für Orthopädie, MediClin Bliestal Kliniken, Blieskastel

\title{
Gelenkinfekt und Arthrofibrose des Kniegelenks
}

Liebe Leserinnen und Leser,

Komplikationen begleiten die Tätigkeit jedes Gelenkchirurgen. Die Qualität eines Operateurs und eines Behandlungsteams misst sich gerade auch an der Beherrschung und Behebung von Komplikationen, seien es solche aus eigener Arbeit oder diejenigen anderer Ärzte.

> Für dieses Heft wurden zwei wesentliche Problemkreise herausgegriffen: der Gelenkinfekt und die Gelenksteife.

Wir hoffen, dass die Beiträge von Lewalter, Schumann und Müller-Rath einen Überblick über den aktuellen Stand hinsichtlich Infektprävention, Infektdiagnostik und -therapie bieten. Hierbei wurde insbesondere auf die Berücksichtigung der spezifischen Belange der arthroskopischen Chirurgie Wert gelegt.

Zur Gelenksteife wird zunächst die Frage der Relevanz von Bewegungseinschränkungen adressiert. Der Automatismus Bewegungshemmung entspricht Arthrofibrose erscheint uns nicht gerechtfertigt. Krenn stellt daher den derzeitigen Stand der histologischen Diagnostik dar, wobei hier v. a. Erfahrungen aus dem Bereich der Endoprothetik einfließen.

Anschließend werden die Möglichkeiten der arthroskopischen und offenen Therapie der Kniesteife dargestellt, wobei zu betonen ist, dass es sich in jedem Fall um Eingriffe für erfahrene Chirurgen handelt. Es ist sicher empfehlenswert, diese Patienten an Kollegen zu verweisen, die sich speziell mit Gelenksteife und Arthrofibrose beschäftigen.
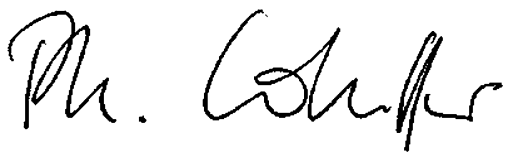

Philipp Lobenhoffer

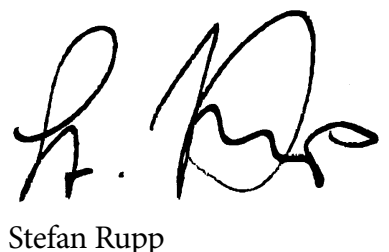

\section{Korrespondenzadresse}

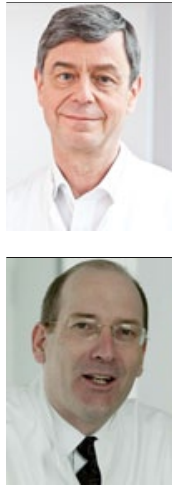

Prof. Dr. P. Lobenhoffer

Sportsclinic Germany Uhlemeyerstrasse 16, 30175 Hannover Philipp.Lobenhoffer@ sportsclinicgermany.com

Prof. Dr. S. Rupp Fachklinik für Orthopädie, MediClin Bliestal Kliniken Am Spitzenberg, 66440 Blieskastel stefan.rupp@mediclin.de

Interessenkonflikt. P. Lobenhoffer und S. Rupp geben an, dass kein Interessenkonflikt besteht. 